\title{
Histerectomía: la experiencia de no tener útero para un grupo de mujeres chilenas
}

\author{
María Teresa Urrutia, PhD $1 a$, Alejandra Araya, PhD 1a, Claudia Flores ${ }^{1 a}$, \\ Daniel Jara 1a, Sergio Silva², María Jesús Lira $3 a$. \\ ${ }^{1}$ Enfermera(o) Matrón(a), Licenciado(a) en Enfermería. ${ }^{2}$ Médico Ginecólogo, Complejo Asistencial Doctor Sotero del \\ Río. ${ }^{3}$ Alumna de Enfermería. a Escuela de Enfermería, Pontificia Universidad Católica de Chile.
}

\section{RESUMEN}

Antecedentes: El útero ha sido descrito como un símbolo de femineidad, sexualidad y reproducción por lo que su extracción conlleva una serie de significados para la mujer. Objetivo: Describir el significado de la histerectomía para un grupo de mujeres sometidas a esta cirugía. Método: Estudio cualitativo realizado en 52 mujeres histerectomizadas. Para la recolección de los datos se utilizaron entrevistas en profundidad y grupos focales. Resultados: Se identificaron 6 dimensiones: síntomas previos, alivio de no tener útero, mitos y creencias, significado del útero y de la cirugía, miedo y sexualidad. Considerar a la histerectomía solamente como tratamiento de una enfermedad sería una visión sesgada de la realidad que vive la mujer, y por lo tanto, alejada de las necesidades que ellas y sus parejas tienen. Conclusión: Los profesionales que intervienen a la mujer deben comprender el proceso complejo que significa para ella asumirse como mujer histerectomizada.

\section{PALABRAS CLAVE: Histerectomía, significado, educación, creencias, mitos, sexualidad}

\section{SUMMARY}

Background: The uterus has been described as a feminine symbol, sexuality and reproduction, therefore the hysterectomy has a multiple meaning by the woman. Aims: To describe the meaning that hysterectomy has by a group of hysterectomized women. Method: Qualitative study with 52 hysterectomized women. By the data collection interviews and focus group was used. Results: It were identified 6 dimensions: previous symptoms, sense of wellness of not having uterus, beliefs and myths, the meaning of uterus and surgery, fear and sexuality. Hysterectomy only like a disease solution would be and slanted vision about the reality that the woman live, and therefore moved away of the needs that they and their partners have. Conclusion: The health care professionals must understand the complex process that means her to assume herself like hysterectomized woman.

\section{KEY WORDS: Hysterectomy, meaning, education, beliefs, myths, sexuality}

\section{INTRODUCCIÓN}

Cada año un gran número de mujeres se ve enfrentada a la extracción del útero (1-5), siendo la histerectomía (HT) una de las cirugías más fre- cuentes después de la cesárea $(6,7)$. La patología benigna del útero es la principal causa de la HT (13 ). Estadísticas a nivel internacional reportan que el $50 \%$ de las mujeres histerectomizadas, además serán sometidas a ooforectomía $(8,9)$. 
La decisión de extraerse el útero es tomada por la mujer tras un largo período con síntomas ginecológicos (10), pudiendo incluso transformarse en la última opción terapéutica (11). En la mayoría de los casos la HT es una cirugía programada (1-3), lo que permite desarrollar una intervención en este grupo de mujeres acorde a sus necesidades.

Para la mujer histerectomizada, la cirugía conlleva una experiencia multidimensional, cuyos efectos evolucionan a lo largo del tiempo $(12,13)$. El útero ha sido descrito como un símbolo de femineidad, sexualidad, reproducción y maternidad (14-18), por lo que la extracción del órgano determina una serie de significados para la mujer. El significado que la mujer otorgue a la HT puede influir directamente en el proceso de toma de decisión de someterse a esta cirugía. Un sentido positivo o negativo sobre esta cirugía puede representar que la mujer acceda tempranamente o retrase el procedimiento.

El objetivo de este estudio es describir el significado de la histerectomía para un grupo de mujeres chilenas con el propósito de contribuir al conocimiento que los profesionales tienen en relación a este tema y sensibilizarlos respecto al significado que tiene para las mujeres.

\section{PACIENTES Y MÉTODO}

Estudio cualitativo, realizado en 52 mujeres histerectomizadas. El estudio contempla entrevistas en profundidad y grupos focales. Del total de mujeres ingresadas al estudio 15 fueron entrevistadas individualmente y 37 participaron en los 4 grupos focales. Ambas metodologías de recolección de datos fueron aplicadas por el equipo investigador entrenado para este propósito. La muestra en estudio es una muestra intencionada de mujeres histerectomizadas cuyos criterios de inclusión fueron: ser usuaria del Complejo Asistencial Dr. Sotero del Río (Santiago, Chile), histerectomía por patología ginecológica benigna durante los 6 meses previos al momento de la recolección de los datos y deseo de participar. El criterio de exclusión fue tener una histerectomía por causa obstétrica. Del listado de mujeres histerectomizadas que cumplieron con los requisitos de inclusión, fueron seleccionadas al azar y contactadas vía telefónica por la investigadora principal para invitarlas a participar. Una vez que accedieron a participar, se les citó a la entrevista o grupo focal según correspondiera.

La recolección de los datos fue realizada entre los meses de mayo a septiembre de 2010. El día de la citación se les solicitó leer y firmar un consentimiento informado, donde se explicó las características del estudio y sus derechos como sujetos de investigación. Posterior a la firma del consentimiento se inició la entrevista o el grupo focal. Tanto las entrevistas como los grupos focales fueron realizadas en una sala privada. Las entrevistas duraron en promedio 40 minutos y los grupos focales 90 minutos.

El inicio de cada entrevista o grupo focal estuvo direccionada por la pregunta: 1) ¿Qué ha significado para usted que le hayan sacado el útero? Las entrevistas y grupos focales fueron grabadas, para posteriormente ser transcritas de manera textual. Se logró la saturación de los datos en la entrevista 10, sin embargo se continuó hasta la entrevista 15 dada la programación previa. Esta investigación contó con la aprobación de los Comités de Ética de la Escuela de Enfermería de la Pontificia Universidad Católica de Chile y del Servicio de Salud Metropolitano Sur Oriente.

Para el análisis de los datos se utilizó la perspectiva fenomenológica descrita por Giorgi (19), realizando el análisis de contenido según Krippendorf (20). Los escritos fueron analizados por 4 investigadores de manera separada utilizando la siguiente metodología: una lectura del escrito sin interpretación por una vez, una segunda lectura para identificar los significados que la mujer manifiesta implícita o explícitamente, cada uno de los significado debía ser respaldado por una frase textual; una vez identificados los significados, éstos fueron agrupados en dimensiones. Una vez identificados los significados y sus respectivas dimensiones, se volvía a leer el escrito para comprobar dicha clasificación. Cada investigador propuso los significados y las dimensiones encontradas de manera independiente, discutiéndose cada propuesta hasta llegar a un consenso.

Para evaluar la credibilidad de los análisis y asegurar la validez descriptiva, se usaron los criterios de Creswell (21). Primero: se utilizó el chequeo de los resultados obtenidos entre las mujeres, después de ser analizadas las entrevistas en profundidad, se realizaron grupos focales para corroborar la validez de los datos obtenidos en las entrevistas. Segundo: se incorporó una descripción detallada de los relatos en cada uno de los significados y dimensiones encontradas, de manera que el lector pudiera determinar su utilidad y aplicabilidad de los resultados de este estudio. Finalmente, el investigador principal examinó los resultados con 3 investigadores expertos en el área, los cuales consideraron válidos el análisis de datos.

\section{RESULTADOS}

El promedio de edad de las mujeres es de 47,4 $\pm 8,3$ años, con rango de 35 a 73 años. El promedio de años de escolaridad fue de 9,7 $\pm 3,1$ años con rango de 3 a 17 años. Del total de mujeres: 39 son casadas, 6 separadas, 6 solteras y 1 viuda. De aquellas que tienen pareja $(n=42)$, el promedio de años de convivencia es de 24,5 \pm 9,5 años con rango entre 7 y 50 años.

La causa más frecuente de HT fue la miomatosis 
uterina. La vía de abordaje de la cirugía fue en 27 casos abdominal, 24 vaginal y en un caso indeterminado, dado que la usuaria afirma que ella aún conserva su útero y que no fue histerectomizada. En 6 casos hubo ooforectomía asociada a la histerectomía, en 35 casos no y en 11 casos las mujeres refirieron no tener información al respecto.

Los relatos de las mujeres nos permiten identificar 6 dimensiones con sus correspondientes temas (Tabla I).

La dimensión "síntomas previos", refiere a aquellas señales físicas propias de una patología ginecológica que es descrita por las mujeres como

Tabla I

\section{DIMENSIONES Y TEMAS EXTRAÍDOS DE LOS RELATOS DE LAS MUJERES HISTERECTOMIZADAS}

\begin{tabular}{ll}
\hline Dimensión & Temas \\
\hline Síntomas Previos & Tipos de síntomas \\
& Latencia de la sintoma- \\
tología \\
Limitación de la actividad \\
de la mujer
\end{tabular}

Alivio de no tener útero No tener síntomas No desarrollar cáncer No tener dificultades sexuales

Mitos y Creencias

Representación del útero y de la cirugía

Miedo

Al cáncer

A la cirugía

A quedar hueca

Al funcionamiento sexual

Sexualidad

Antes de la histerectomía La primera relación sexual Después de la histerectomía una vivencia relevante previo a ser histerectomizada. Junto con la descripción de los síntomas (reglas abundantes, incontinencia, dolor y anemia), las mujeres además relatan la latencia de estos síntomas por periodos prolongados antes de la extracción del útero y como estos síntomas les provoca limitación de las actividades de la mujer.

Mujer: "...y cuando me llegaban en esos tres meses la regla era como, era como abrir una llave, aparte de eso que botaba así como pana, y yo no entendía por qué, yo decía ¿por qué me está sucediendo esto?, ya no, mi cuerpo ya no tenía fuerzas, si caminaba una cuadra me cansaba..."

Mujer: "La primera vez vieron mi caso porque venía solamente para que me sacaran los miomas y el doctor dijo que no, que me tenía que hacer una histerectomía... entonces ya esperar un año más, desde febrero del 2009 a marzo del... del 2010 que me llamaron para operarme, así que fue bien larga la espera y todos esos años con sangramiento, dolor y también lo mismo que decían...nada de marido o sea... por allá el pobre".

Mujer: “...porque yo quería vivir una vida más ...dijera más agradable, mejor ¿me entiende usted? porque uno anda así, claro anda súper mal, y me sentía horrible, no podía salir a la calle...si salía tenía que volver ligerito, tenía que ponerme pañales entonces ...es recomplicado y acercarse a alguien que este olor a pichi (orina) es súper desagradable entonces yo no vivía una vida muy, muy grata para decirle francamente".

El "alivio de no tener útero" es una dimensión que refleja la sensación de libertad que las mujeres describen por el cese de los síntomas físicos, por el fin de los problemas sexuales pre quirúrgicos y por la tranquilidad de no desarrollar un cáncer.

Mujer: “...en parte de que no me llegue la regla para mi es una alegría porque ya no estoy preocupada de todos los meses estar con mi regla, a veces que eran molestas, que dolorosas, entonces en esa parte es bueno".

Mujer: "Para mí fue bueno, fue bueno, me alivió en el sentido de mi relación con mi pareja, porque yo no podía tener relación por los dolores, ya no los tengo, no he tenido dolores y... no, nos ha servido bastante a los dos, a los dos nos ha servido".

Mujer: “...yo lo único que quería era que me sacaran luego el útero para que no me pasara a cáncer o sea que no produ... no se produciera un cáncer"

La experiencia de no tener útero se encuentra rodeada de "mitos y creencias" en torno a esta cirugía. El quedar "hueca", dejar de ser mujer o tener problemas con la pareja como consecuencia de la $\mathrm{HT}$, son los comentarios que las mujeres deben 
escuchar y enfrentar durante el proceso quirúrgico. Dichos mitos generan dudas y dolor en la mujer.

Mujer: "...entonces es tanto lo que le ...le colocan la gente, las amistades en la cabeza, que le dicen que queday hueca, que al tener relaciones no es lo mismo, es como que estay vacía, como que soy un cajón vacío...".

Mujer: "...entonces yo creo que se ha visto siempre como que...pucha la mujer como que tiene que estar completita... pero a la mujer como que siempre se le exige estar completa y como que si le falta una parte del cuerpo ya no es mujer, ...como que no funciona en ese sentido, se ve, se ha visto desde siempre...desde chica a uno le dicen".

Mujer: Porque es él el que me lo averigua a mí, me dice no tú estás mal, no tenís que hacerle caso... yo igual sí, yo encuentro que... pero igual a veces el poco tino como le digo de la gente a uno le afecta cuando está recién operada y todo, uno está sensible igual po'.

Mujer: "...también hay personas que...que te dicen o sea tu lo comentas ...me voy a operar me van a sacar el útero y lo primero que la gente te dice a vai a quedar hueca, o no vai a tener... no vas a poder tener relaciones, o vas a tener y no vai a sentir ...y es muchísima la gente que tiene esa idea y que uno la escucha... yo sabía que no era así, porque la doctora me lo había explicado a mi, pero uno igual lo escucha... igual uno queda como ... si tanta gente lo dice ...".

La "representación del útero y la cirugía" es otra dimensión identificada en los relatos de las mujeres. El útero es señalado como un órgano asociado a la sexualidad de pareja y al rol materno; a su vez la histerectomía es una cirugía que debe mantenerse en el ámbito privado, afectando la imagen femenina y provocando en la mujer una sensación de vacío, de quedar "hueca" o de que algo les falta.

Mujer: "... bueno si me sacaron un órgano, algo extraño tiene que haber adentro o sea... no puede ser que ya todo esté normal, entonces el cuerpo ya... ya está sin un órgano, entonces queda la preocupación de... bueno ya, por mí no importa porque yo sigo haciendo mi vida igual, actúo igual, todo... pero mi pareja ¿qué va a sentir él? ...¿cómo va a reaccionar él?".

Mujer: "...porque en ese momento me vino como, como depresión...porque ya uno no es lo mismo, de cuando uno se enferma, uno ya sabe que va a llegar su regla, sabe que puede engendrar, pero ya después no...".

Mujer: "... no ando diciéndole a todo el mundo yo no tengo útero... son cosas que... igual mis amigas saben que no tengo útero pero son cosas que... son como muy para dentro para uno"'.
Mujer: "pero yo mi tema fue tan personal, que yo y mi familia sabia de lo que yo me iba a operar... nadie más, ...por lo menos para yo evitarme tener que darle explicaciones a la gente, que la gente comentara, porque eso era lo que yo no quería o sea yo creo que a mi lo que me costó más aceptar fue eso, que los demás no comentaran lo que a mi me estaba pasando".

Mujer: "...tengo la esperanza de que si... con el tiempo, como toda operación los ... los tejidos se tienen que volver a regenerar y todo eso... yo digo a lo mejor, después voy a ser una mujer plena, igual que antes... como antes de la operación, pero con el tiempo, pienso yo... pero como que algo falta, no sé qué es pero algo falta"

El "miedo" logra crear una propia dimensión en los relatos de las mujeres. El temor que la mujer describe es al posible diagnóstico de cáncer (en caso que no se hubiesen extraído el útero), a la cirugía y anestesia, y a las posibles consecuencias de la HT tanto en la sensación de quedar hueca o vacía como en el ámbito sexual.

Mujer: "...antes de operarme, era el miedo no sé a que tenía... cáncer, cáncer, cáncer...”.

Mujer: “...y la matrona me dijo que era urgente, el médico también le puso urgente ... me dio miedo, me dio miedo... dejé pasar ... varios meses...varios meses, como tres meses.... hasta que me llamó la matrona, y dijo, le doy la última oportunidad ... me dijo, ¿viene?... o borro la ficha.... Voy le dije yo".

Mujer: "....pero sí tuve temor al principio, a pesar de todos los conocimientos que yo tenía, de que iba a encontrar un hueco, como que iba a encontrar un vacío... y me daba miedo eso".

Mujer: "...por ser yo siempre decía... no, no, no, no todavía no puedo porque tengo que ir al doctor... siempre le metía chivas (disculpas) a mi marido, entonces un día le dije a la matrona le dije... fui y le digo yo ¿sabe qué señorita?...que me da miedo, yo le dije a ella ...sabe me da mucho miedo tener relaciones porque yo creo que estoy vacía por dentro".

La "sexualidad" cobra una dimensión propia en los relatos de las mujeres histerectomizadas. La descripción de la vida sexual afectada por la enfermedad antes de la cirugía, como de los cambios posteriores son dos de los tres temas identificados en esta dimensión. La primera relación sexual es el tercer tema que cobra relevancia en el relato de las mujeres.

Mujer: “....y yo me sentía súper mal porque, no podía satisfacer a mi esposo...con la parte relación sexual porque ellos como uno también necesitan y yo no podía hacerlo por la situación que me sentía tan mal y que no estaba bien de mi interior... entonces yo antes de la operación tuve muchas complicaciones...". 
Mujer: "....y la ansiedad después de probar, eso es lo que más yo creo que también me marcó, de saber...el médico me dijo después de 2 meses sin relaciones, y yo esperé casi $4 . .$. y de ahí yo ... era de saber, si iba a volver a acabar (orgasmo), si iba a volver a sentir, era sensación, era no sé, igual que un niño, que cómo va funcionar y felizmente todo bien".

Mujer: "...noté antes de la operación, yo no tenía, ni una gana, nada de nada nada de estar con mi marido, nada, cero, yo decía pobrecito, y no me daban ganas no más, pero ahora ya, después de la operación, hemos tenido, dos, dos cercanía y ha resultado, que yo encuentro que es una maravilla, comparado con todo lo anterior...".

\section{DISCUSIÓN}

La principal fortaleza de este estudio es la utilización de 2 técnicas de recolección de datos cualitativos (entrevistas en profundidad y grupos focales) y la confluencia en ambos grupos de estudio (entrevistas y grupos focales) de las mismas dimensiones descritas en relación al significado de la cirugía en mujeres histerectomizadas. Mediante una aproximación cualitativa se logró extraer la vivencia de la mujer permitiendo entender el fenómeno desde una perspectiva amplia y profunda. La principal limitación es que considera solo la vivencia de mujeres en los primeros 6 meses post HT. Sería interesante conocer cuál es la percepción que tienen las mujeres una vez transcurrido más tiempo post operadas.

La sensación de alivio y, por lo tanto, de sentirse mejor posterior a la HT coincide con reportes previamente publicados $(5,17,18,22)$. El alivio se puede relacionar a la presencia de síntomas previos a la cirugía, la que según los relatos de las mujeres son de larga data y alteran profundamente la vida cotidiana de cada una de ella, situación presente en otros estudios también $(8,22)$.

El significado que da la mujer al útero claramente trasciende el entorno cultural en el cual se encuentra. El útero como un órgano que da vida, asociado al rol de ser madre ha sido ampliamente reportado en otras culturas $(14,16)$. Por otro lado, y respecto a la sensación de "dejar de ser mujer", en un estudio cuantitativo realizado en EEUU encontró que la imagen de la mujer respecto de su femineidad se vio afectada sobre todo a edades menores (23). Al respecto Rivera y cols (16), señalan producto de los hallazgos de su estudio: "Ser mujer es tener útero, y tener útero es ser femenina", reafirmando un concepto arraigado en la población y cuestionado desde hace muchas décadas. Simone de Beavoir (24), en la introducción de su libro "el segundo sexo" escrito en la década del 40 , ya hacía referencia al concepto de ser mujer como "tota mulier in utero" (toda mujer es útero), señalando posteriormente que la relación con el órgano no la hace mujer, y que la feminidad es una realidad misteriosa de la cual la mujer participa. La asociación del útero al rol femenino dado que está arraigado en el inconsciente colectivo, debe ser tratado con delicadeza al momento de educar a la mujer sometida a esta cirugía, relevando el rol femenino a algo que va mas allá de poseer o no el órgano en cuestión. Es de importancia educar a las mujeres y sus parejas que: no tener útero no es sinónimo de no ser mujer.

Los problemas con la pareja forman parte de las creencias asociadas a la extracción del útero como también a los miedos relacionados a las consecuencias de dicha cirugía (14). En un estudio brasileño (16), la relación conyugal fue descrita como un aspecto que causa dudas en la mujer, sobre todo en lo referente al ámbito sexual. Al respecto, es necesario integrar al proceso educativo a la mujer y su pareja, por la gran relevancia que el hombre tiene como soporte emocional. Si el hombre participa del proceso, muchos de los miedos pueden ser erradicados y las creencias de abandono ser descartadas. Cabe señalar que en un estudio chileno (25), el $100 \%$ de las mujeres refiere que le gustaría que su pareja fuese incorporado a la educación sobre su cirugía.

El miedo ha sido descrito previamente por otros autores en otros países (23). En este estudio, el miedo por un lado se asocia al evento quirúrgico propiamente tal, por otro y con más fuerza se presenta asociado a las consecuencias de la cirugía $(14,26)$. Augustus $(17)$, en un estudio etnográfico encontró, al igual que este estudio, que el miedo de la mujer se relaciona al funcionamiento sexual postoperatorio. Es importante señalar que si la mujer conociera más sobre su anatomía, patología y el efecto que la HT tiene, podría disminuir el temor, enfrentando la cirugía de manera más apropiada. El miedo en su mayoría se relaciona al desconocimiento del proceso quirúrgico y de la anatomía genital femenina.

La HT ha sido descrita como un factor de riesgo, sobre todo en mujeres jóvenes, para desarrollar depresión $(23,27)$ y para tener una peor calidad de vida posterior a la cirugía (23). Cabe señalar que existen vivencias descritas en el presente estudio que podrían relacionarse al desarrollo de un cuadro depresivo como son el miedo, la sensación de vacío, las creencias, los problemas en el ámbito de pareja, la redefinición del rol a cumplir como mujer 
sin útero. Se debe por lo tanto estar atenta a la aparición de síntomas depresivos en la mujer para su rápida y oportuna referencia a especialista.

La presencia de ooforectomía asociada a la histerectomía es un aspecto que debe ser considerado. En un estudio previo realizado en Chile, la satisfacción sexual de las mujeres histerectomizadas mejoró significativamente a los 6 meses post cirugía (28), sin embargo, en el mismo grupo de estudio la presencia de ooforectomía fue descrito como un factor diferenciador de las características de sexualidad, señalando que algunos parámetros se ven alterados en el grupo con HT más ooforectomía (29), no así en el grupo de mujeres histerectomizadas solamente. Por otro lado, en un estudio realizado en Inglaterra se señaló que aquellas mujeres con ooforectomía presentaron mayor severidad en los síntomas que aquellas que conservaron sus ovarios (30). Por lo anterior es importante al momento de educar a la mujer explicar la diferencia entre ambas cirugías, y que no todas las histerectomías consideran además la extracción ovárica. Cabe señalar que en el presente grupo de estudio, hubo 11 mujeres que desconocían si habían sido o no ooforectomizadas.

Finalmente, una reflexión sobre la importancia que tienen las investigaciones en esta área para poder entregar información respecto del impacto a nivel individual que tiene la cirugía. Si bien no hay duda que la HT es el tratamiento adecuado para las mujeres con ciertas patologías ginecológicas, lo que se refleja en los relatos de las mujeres de las dos primeras dimensiones identificadas en este estudio, es necesario también reconocer que esta cirugía se transforma paralelamente, al alivio y cese de síntomas, en una problemática en el fuero más íntimo de la mujer y de su relación de pareja. La mujer reconoce que la experiencia de ser histerectomizada es "privada", y que solo comparte con aquellos seres más cercanos, por ende, el miedo, las dudas no resueltas, la sensación de vacío y de no ser mujer, son aspectos que la acompañan posterior a la cirugía y que si no son valorados adecuadamente por el profesional de salud que las atiende, difícilmente podrán ser resueltos por la propia mujer y su pareja. Si a lo anterior se agrega la transmisión cultural de mitos y creencias erradas en torno a la HT, se produce un círculo vicioso que perpetua los sentimientos negativos y por ende la desinformación al respecto.

\section{CONCLUSIÓN}

Es necesario que el profesional de salud asuma un rol activo al momento de intervenir a la mujer, desmitificando las creencias y entregándoles una educación que les permita enfrentar dichas dificultades. Considerar a la HT solamente como un alivio de los síntomas y por ende un tratamiento de la enfermedad, sería una visión sesgada de la realidad que vive la mujer, y por lo tanto alejada de las necesidades que ellas y sus parejas tienen. El llamado es a intervenir en los aspectos biomédicos y psicosociales comprendiendo el proceso complejo que significa para ella asumirse como mujer histerectomizada.

\section{REFERENCIAS}

1. Brill Al. Hysterectomy in the 21st century: different approaches, different challenges. Clin Obstet Gynecol 2006;49:722-35.

2. Katz A. Sexuality after hysterectomy: a review of the literature and discussion of nurses' role. J Adv Nurs 2003;42:297-303.

3. Chou CC, Lee TY, Sun CC, Lin SS, Chen LF. Husbands' experiences before wives' hysterectomy. J Nurs Res 2006;14:113-22.

4. Skea Z, Harry V, Bhattacharya S, Entwistle V, Williams B, MacLennan G, et al. Women's perceptions of decision-making about hysterectomy. BJOG 2004;111:133-42.

5. Fleming V. Hysterectomy: a case study of one woman's experience. J Adv Nurs 2003;44:575-82.

6. Brown JS, Sawaya G, Thom DH, Grady D. Hysterectomy and urinary incontinence: a systematic review. Lancet 2000;356:535-9.

7. Thakar R, Ayers S, Clarkson P, Stanton S, Manyonda I. Outcomes after total versus subtotal abdominal hysterectomy. N Engl J Med 2002;347:1318-25.

8. Lindberg CE, Nolan, LB. Women's decision making regarding hysterectomy. J Obstet Gynecol Neonatal Nurs 2001;30:607-17.

9. Sharts-Hopko NC. Histerectomy for nonmalignant conditions. Am J Nurs 2001;101:32-40.

10. Williams R, Clark A. A qualitative study of women's hysterectomy experience. J Womens Health Gend Based Med 2000;9:33-42.

11. Kuppermann $M$, Varner RE, Summitt RL Jr, Learman LA, Ireland C, Vittinghoff E, Stewart AL, Lin F, Richter HE, Showstack J, Hulley SB, Washington $A E$. Effect of hysterectomy vs medical treatment on health-related quality of life and sexual functioning: the medicine or surgery (Ms) randomized trial. JAMA 2004;24;291:1447-55.

12. Katz A. Sexually speaking. Sexually and hysterectomy: finding the right words. Am J Nurs 2005;105:65-8.

13. Wade J, Pletsh PK, Morgan SW, Menting SA. Hysterectomy: what do women need and want to know? J Obtest Gynecol Neonatal Nurs 2000;29:33-42.

14. Reis N, Engin R, Ingec M, Bag B. A qualitative study: beliefs and attitudes of women undergoing abdominal hysterectomy in Turkey. Int $\mathrm{J}$ Gynecol Cancer 2008;18:921-8. 
15. Groff JY, Mullen PD, Byrd T, Shelton AJ, Lees E, Goode J. Decision making, beliefs, and attitudes toward hysterectomy: a focus group study with medically underserved women in Texas. J Womens Health Gend Based Med 2000;9 Suppl 2:S39-50.

16. Rivera A, Martins M, Bedone A. O significado da retirada do utero para as mukheres: um estudo qualitativo. Rev Assoc Med Bras 2005;51:270-4.

17. Augustus CE. Beliefs and perceptions of African American women who have had hysterectomy. J Transcult Nurs 2002;13:296-302.

18. Galavotti C, Richter DL. Talking about hysterectomy: the experiences of women from four cultural groups. J Womens Health Gend Based Med 2000;9 Suppl 2:S63-7.

19. Giorgi A. Sketch of a psychological phenomenological method. In: Aanstoos CM, Fischer WF, Giorgi A, Wertz FJ, editors. Phenomenology and Psychological Research Pittsburgh: Duquesne University Press; 1985.

20. Krippendorf K. Content Analysis. An introduction to its methodology. Thousands Oaks: Sage Publications; 2004.

21. Creswell JW. Standards of validation and evaluation. Qualitative inquiry and research design: choosing among five approaches. 2 ed. Thousand Oaks, CA: Sage Publications; 2007. p. 201-21.

22. Mingo C, Herman C, Jasperse M. Women's Stories: Ethnic variations in women's attitudes and experiences of menopause, hysterectomy, and hormone repla- cement therapy. J Womens Health Gend Based Med 2000;9 Suppl 2:27-38.

23. Cabness J. The psychological dimensions of hysterectomy: Private places and the inner spaces of women at midlife. Soc Works Health Care 2010;49:211226.

24. De Beauvoir S. Introducción. En: De Beauvoir S (Ed). El segundo sexo. Sexta ed. Buenos Aires: Ediciones de Bolsillo; 2010. p 15.

25. Urrutia MT, Riquelme P, Araya A. Educación de mujeres histerectomizadas: ¿qué desean saber? Rev Chil Obstet Ginecol 2006;71:410-6.

26. Marvan ML, Trujillo P, Karam MA. Hysterectyomy as viewed by Mexican women and men. Sex Roles 2009;61:688-98.

27. Mantani A, Yamashita H, Fujikawa T, Yamawaki S. Higher incidence of hysterectomy and oophorectomy in women suffering from clinical depression: retrospective chart review. Psychiatry Clin Neurosci 2010;64:95-8.

28. Urrutia MT, Araya A, Villarroel L, Vinales D. Características y evolución de la sexualidad en mujeres histerectomizadas. Rev Chil Obstet Ginecol 2004;69:301-6.

29. Urrutia MT, Araya A, Padilla O. Sexualidad e histerectomía: diferencias entre un grupo de mujeres con y $\sin$ ooforectomía. Rev Chil Obstet Ginecol 2011;76:13846.

30. Bhavnani V, Clarke A. Women awaiting hysterectomy: a qualitative study of issues involved in decisions about oophorectomy. BJOG 2003;110:168-74. 